\title{
Endometrioid Stromal Sarcoma of the Vagina
}

National Cancer Institute

\section{Source}

National Cancer Institute. Endometrioid Stromal Sarcoma of the Vagina. NCI Thesaurus.

Code $C 40270$.

A rare sarcoma that arises from the vagina. This category includes low grade endometrioid stromal sarcoma and undifferentiated vaginal sarcoma. 